\title{
Three unsuspected CT diagnoses of COVID-19
}

\section{David $\mathrm{Vu}^{1} \cdot$ Maryanne Ruggiero ${ }^{1} \cdot$ Woo Sung Choi $^{1} \cdot$ Daniel Masri $^{1} \cdot$ Mark Flyer $^{1} \cdot$ Inna Shyknevsky $^{1} \cdot$ Evan G. Stein $^{1}$}

Received: 19 March 2020 / Accepted: 30 March 2020 / Published online: 13 April 2020

(C) American Society of Emergency Radiology 2020

\begin{abstract}
Purpose Coronavirus disease 2019 (COVID-19) is caused by a novel strain of coronavirus named severe acute respiratory syndrome coronavirus 2 (SARS-CoV-2) that has quickly spread around the globe. Health care facilities in the USA currently do not have an adequate supply of COVID-19 tests to meet the growing demand. Imaging findings for COVID-19 are nonspecific but include pulmonary parenchymal ground-glass opacities in a predominantly basal and peripheral distribution. Methods Three patients were imaged for non-respiratory-related symptoms with a portion of the lungs in the imaged field. Results Each patient had suspicious imaging findings for COVID-19, prompting the interpreting radiologist to suggest testing for COVID-19. All 3 patients turned out to be infected with COVID-19, and one patient is the first reported case of the coincident presentation of COVID-19 and an intraparenchymal hemorrhage.

Conclusion Using imaging characteristics of COVID-19 on abdominal or neck CT when a portion of the lungs is included, patients not initially suspected of COVID-19 infection can be quarantined earlier to limit exposure to others.
\end{abstract}

Keywords COVID-19 · Ground-glass opacities · Intraparenchymal hemorrhage

\section{Introduction}

Coronavirus disease 2019 (COVID-19) is caused by a novel strain of coronavirus called severe acute respiratory syndrome coronavirus 2 (SARS-CoV-2) that was first discovered in Wuhan, China, in December 2019. The virus has quickly spread around the globe and has been declared a global pandemic by the World Health Organization. As of March 15, 2020, there are 153,517 confirmed cases of COVID-19 in 49 states, 140+ countries, and on 6 continents [1, 2]. In the USA, hospitals, clinics, nursing homes, and other health care facilities currently do not have an adequate supply of COVID-19 real-time polymerase chain reaction (RT-PCR) assays to meet the demand for possible cases.

An increasing number of confirmed patients in the USA have now tested positive without a travel history or known exposure to a confirmed COVID-19 patient. Imaging findings for COVID-19 are non-specific and variable but most commonly show rounded pulmonary parenchymal ground-glass opacities without lung cavitation, discrete pulmonary nodules,

Evan G. Stein

egstein@maimonidesmed.org

1 Radiology Department of Maimonides Medical Center, 4802 10th Ave, Brooklyn, NY 11219, USA or pleural effusions [3]. Other less common imaging characteristics include bronchial wall thickening, linear opacities, crazy paving pattern, and the reverse halo sign. Patients imaged within the first 2 days of being infected can also show normal chest $\mathrm{CT}$ findings [4].

Here, we present a series of 3 patients imaged for nonrespiratory-related symptoms with a portion of the lungs in the imaged field. Each patient had suspicious imaging findings for COVID-19, which prompted the interpreting radiologist to suggest that the clinical team test for COVID-19.

Ultimately, all 3 patients were found to be infected with COVID-19, and one patient is the first reported case of the coincident presentation of COVID-19 and an intraparenchymal hemorrhage.

\section{Case 1}

A 69-year-old man with a history of nephrolithiasis presented to the Emergency Department (ED) on March 6, 2020, complaining of generalized body aches and suprapubic pain. He had returned from a trip to Egypt 10 days earlier and developed these symptoms a week prior to presentation. The patient denied sick contacts, fever, chills, fatigue, cough, or shortness of breath. 
Fig. 1 Axial images (a and $\mathbf{b}$ ) from a contrast-enhanced CT of abdomen and pelvis through the lung bases show several groundglass opacities bilaterally

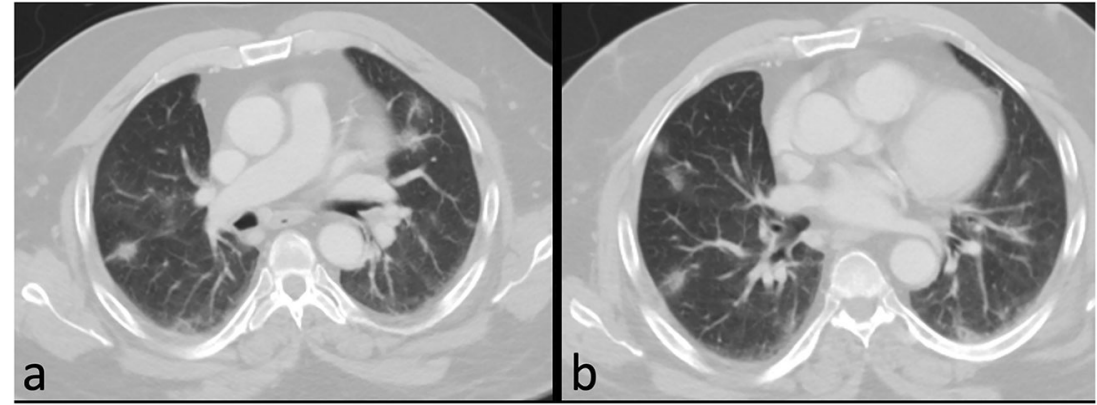

A contrast-enhanced CT of the abdomen and pelvis was performed, and in addition to multiple stones in the left kidney and left ureterovesical junction measuring up to $1.0 \mathrm{~cm}$, the lung bases showed new development of bilateral ground-glass and nodular opacities (Fig. 1). Because of the imaging features and the patient's travel history, COVID-19 was suggested by the radiologist despite the lack of fever and respiratory symptoms.

After consultation with the Department of Health (DOH), there was a low suspicion for COVID-19, but COVID-19 RT-PCR viral swabs were sent to the DOH lab for testing.

The patient was deemed stable for discharge with tamsulosin, pain medication, and an outpatient urology appointment. Instructions were also given for the patient to self-quarantine until the results for COVID-19 came back.

On March 9, 2020, the patient was found to be infected with COVID-19 and told to isolate himself as best as he could in his home.

\section{Case 2}

A 67-year-old man presented to the ED on March 8, 2020, with a chief complaint of increased urinary frequency and right lower quadrant abdominal pain radiating to the pelvis for the past week. The patient's son and niece were sick with unknown illnesses. He had not had any recent travel or exposure to patients with known COVID-19.

A contrast-enhanced CT of the abdomen and pelvis was performed to assess for appendicitis and diverticulitis. Imaging showed no acute abdominal or pelvic pathology, but there were round ground-glass opacities at the lung periphery in the visualized lung bases (Fig. 2). The radiologist suggested COVID-19 as an etiology, and the patient was tested and sent home to quarantine in his home with the approval of the DOH.

Two days later, the patient was notified that he had tested positive for COVID-19 and was directed to remain isolated and to return to the emergency room if any acute respiratory symptoms developed.

\section{Case 3}

A 30-year-old man was transferred to our ED from an outside hospital for neurologic consultation on March 11, 2020. The patient had presented with dysarthria, right hemiparesis, and a right facial droop. A noncontrast head CT performed at the outside hospital showed a $1.7-\mathrm{cm}$ acute left basal ganglia hemorrhage (Fig. 3a). The patient was admitted to the Stroke Service who managed the patient by obtaining a CT angiogram of the head and neck. There was no vascular malformation or other etiology for the hemorrhage, but there were subcentimeter ill-defined nodules in both upper lobes of the lung and a $1.8-\mathrm{cm}$ nodule at the periphery of the left upper lobe (Fig. 3 b and c), and COVID-19 was suggested by the interpreting neuroradiologist.

The patient had no fever, known underlying medical conditions, sick contacts, or recent travel. After additional imaging, including a brain MRI, brain MRA, chest CT, and echocardiogram, the patient tested positive for COVID-19 and was placed in isolation.

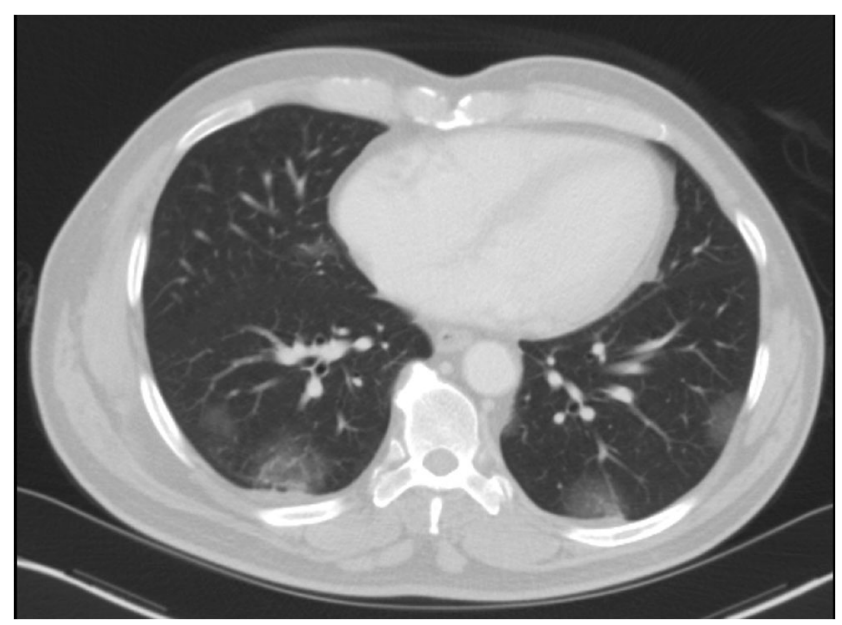

Fig. 2 Axial image from a contrast-enhanced CT of abdomen and pelvis through the lung bases display round ground-glass opacities at the periphery 


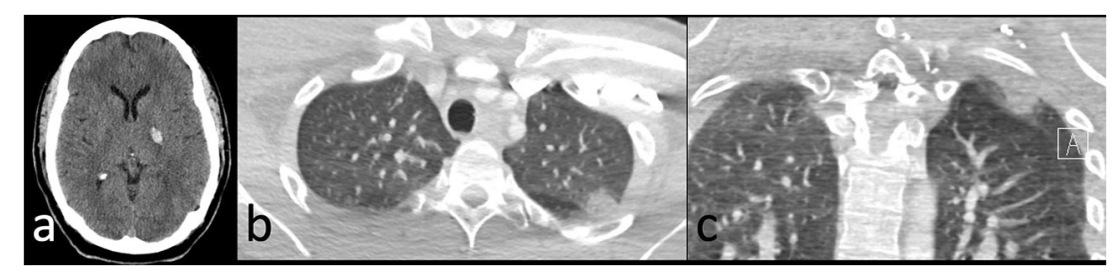

Fig. 3 Axial non-contrast head CT (a) shows a left basal ganglia hemorrhage. Axial (b) and coronal (c) reconstructed images from a CT angiogram of the head and neck through the upper lungs shows a nodule at the periphery of the left upper lobe

\section{Discussion}

With COVID-19 spreading quickly across the globe, the RTPCR tests are not readily available in some communities to meet the demand for the exposed patient population. As of March 10, 2020, the turnaround time for the COVID-19 RTPCR assay was 12-72 $\mathrm{h}$ [5]. One of the earliest publications on COVID-19 and CT imaging found that chest CT had a sensitivity of $98 \%$ for early presentation of COVID-19 compared with $71 \%$ using the RT-PCR assay. The lower sensitivity for RT-PCR found in this study may be due to improper sampling, low viral load at the time of testing, and various detection rates for different manufacturers [6]. While, as of March 22, 2020, the American College of Radiology does not recommend using chest $\mathrm{CT}$ as a screening tool for COVID-19 [7], in patients with possible exposure to COVID-19 or suspicious symptoms, the RT-PCR assay may be used in conjunction with the chest CT findings to triage patients requiring hospitalization from spreading the infection to the vulnerable hospital patient population and frontline health care workers.

In this case series, three patients were imaged without any of the three most common COVID-19 symptoms that include fever, cough, and shortness of breath [8]. However, in each case, a portion of the lungs was scanned, which incidentally yielded suspicious findings and prompted the radiologist to suggest the patients' team test for COVID-19.

It is notable that our 30-year-old patient had coincident intraparenchymal hemorrhage and COVID-19. In 2012, a 42-year-old female patient with Middle East respiratory syndrome coronavirus (MERS-CoV) spontaneously developed both right frontal lobe intraparenchymal and subarachnoid hemorrhages causing midline shift and subfalcine herniation [9]. Our patient presented with a much smaller left basal ganglia hemorrhage, but in this young patient, no etiology was identified. In a recent non-peer-reviewed retrospective study of 214 hospitalized patients, $36.4 \%$ of patients with COVID-19 had neurologic symptoms with the most common being dizzy and headache, as well as 6 with ischemic strokes and 1 with an unspecified hemorrhagic stroke [10]. Between these findings and our encounter with a patient with a basal ganglia hemorrhage, it will be important to pay closer attention to neurologic findings in COVID-19 patients in the future.
Using pulmonary parenchymal ground-glass or nodular opacities in a predominantly peripheral distribution on abdominal or neck CT when a portion of the lungs is included, patients not initially suspected of COVID-19 can be placed into isolation at the hospital earlier and be instructed to stay quarantined until the test results come back, limiting exposure to others. Precautions can be discontinued when the patient has two consecutive negative tests from the nasopharynx and oropharynx more than $24 \mathrm{~h}$ apart [11].

\section{Compliance with ethical standards}

Conflict of interest The authors declare that they have no conflict of interest.

\section{References}

1. Situation Report - 55: https://www.who.int/docs/default-source/ coronaviruse/situation-reports/20200315-sitrep-55-covid-19.pdf? sfvrsn=33daa5cb_8 [Accessed on March 15, 2020]

2. https://www.cdc.gov/coronavirus/2019-ncov/cases-in-us.html [Accessed on March 15, 2020]

3. Chung M, Bernheim A, Mei X et al (2020) CT imaging features of 2019 novel coronavirus (2019-nCoV). Radiology. https://doi.org/ 10.1148/radiol.2020200230

4. Bernheim A, Mei X, Huang M et al (2020) Chest CT findings in coronavirus disease-19 (COVID-19): relationship to duration of infection. Radiology. https://doi.org/10.1148/radiol/2020200463

5. https://www.cdc.gov/coronavirus/2019-ncov/lab/testinglaboratories.html [Accessed on March 12, 2020]

6. Fang Y, Huangqi Z, Xie J et al (2020) Sensitivity of chest CT for COVID-19: comparison to RT-PCR. Radiology. https://doi.org/10. 1148/radiol.2020200432

7. ACR Recommendations for the use of Chest Radiography and Computed Tomography (CT) for Suspected COVID-19 Infection - https://www.acr.org/Advocacy-and-Economics/ACR-PositionStatements/Recommendations-for-Chest-Radiography-and-CTfor-Suspected-COVID19-Infection [Accessed on March 25, 2020]

8. Chen N, Zhou M, Dong X, Qu J, Gong F, Han Y, Qiu Y, Wang J, Liu Y, Wei Y, Xia J, Yu T, Zhang X, Zhang L (2020) Epidemiological and clinical characteristics of 99 cases of 2019 novel coronavirus pneumonia in Wuhan, China: a descriptive study. Lancet. 395:507-513. https://doi.org/10.1016/S0140-6736(20) 30211-7

9. Al-Hameed FM (2017) Spontaneous intracranial hemorrhage in a patient with Middle East respiratory syndrome corona virus. Saudi Med J 38:196-200. https://doi.org/10.15537/smj.2017.2.16255 
10. Mao L, Wang M, Chen S et al (2020) Neurologic manifestations of hospitalized patients with COVID-19 in Wuhan, China: a retrospective case series study. medRxiv. https://doi.org/10.1101/2020.02. 22.20026500
11. Centers for Disease Control and Prevention. Interim Considerations for Disposition of Hospitalized Patients with 2019-nCoV Infection. https://www.cdc.gov/coronavirus/2019-ncov/hcp/dispositionhospitalized-patients.html [Accessed on March 12, 2020]

Publisher's note Springer Nature remains neutral with regard to jurisdictional claims in published maps and institutional affiliations. 